\title{
Perspectives for gallotannins neuroprotective potential - current experimental evidences
}

\author{
Radosław Kujawski', Małgorzata Kujawska², Marcin Ożarowski ${ }^{3,4}$, Justyna Baraniak4, \\ Halina Laskowska', Tamara Nowocień', Magdalena Borowska', Michał Szulc', \\ Agnieszka Sobczak ${ }^{5}$, Przemysław Mikołajczak ${ }^{1,4}$ \\ ${ }^{1}$ Department of Pharmacology, Poznan University of Medical Sciences, Poland \\ ${ }^{2}$ Department of Toxicology, Poznan University of Medical Sciences, Poland \\ ${ }^{3}$ Department of Pharmaceutical Botany and Biotechnology, Poznan University of Medical Sciences, Poland \\ ${ }^{4}$ Department of Pharmacology and Phytochemistry, Institute of Natural Fibers and Medicinal Plants, Poland \\ ${ }^{5}$ Department of Pharmaceutical Chemistry, Poznan University of Medical Sciences, Poland
}

\section{ABSTRACT}

Gallotannins are class of hydrolyzable tannins consisting of gallic acid and a sugar moiety. Currently, there is growing interest around a possible neuroprotective effect of this class of phytochemicals, which is suggested to be a result of their active metabolites. Evidence from experimental studies has suggested that tannin-rich plant preparations might be effective at reversing neurodegenerative pathology and age-related declines in neurocognitive performance. This mini-review summarizes, based on experimental studies, current knowledge about diverse neuroprotective abilities of gallotannins, mostly via antioxidant properties and some mechanisms of the effect are proposed including blocking accumulation of nitrites, inhibiting expression and activity of heme oxygenase 1(HO-1), and decreasing degradation of poly(ADP-ribose) glycohydrolase (PARP).

Keywords: neuroprotection, plant extract, gallotannins, galloylated cyanogenic glycosides, poly(ADP-ribose) glycohydrolase, 1,2,3,4,6-penta-0-galloyl- $\beta$-D-glucopyranose.

\section{Introduction}

In the last decades, numerous studies on a protective role of plant polyphenols against several chronic diseases, including neurodegeneration, have been published [1, 2]. Currently, substantial interest in health benefits of tannins is emerging, especially regarding their neuroprotective potential [3, 4].

Tannins are a subclass of naturally occurring polyphenols found in both condensed and hydrolyzable forms. Hydrolysable tannins are multiple esters of a sugar moiety and organic acids such as gallic acid in gallotannins or ellagic acid in ellagitannins. Instead, condensed tannins occur as oligomerics or polymerics of flavan-3-ols, mainly derivatives of epicatechin and catechin. Tannins have been reported to exert several biological effects, including antioxidant and free radical scavenging activity as well as antimicrobial, anti-cancer, and cardio-protective properties [5]. Evidence from epidemiological and human intervention studies and animal studies have suggested that tannin-rich plants might be effective at reversing neurodegenerative pathology and age-related declines in neurocognitive performance [3-5]. Despite a great volume of literature data showing various biological effects of polyphenols, including tannins, it is still a matter of debate because of their questionable bioavailability. Most of them are poorly absorbed through the gastrointestinal tract, highly metabolised, and rapidly eliminated [6]. There is also contradictory evidence as to whether polyphenolics may cross the blood-brain barrier or not. How- 
ever, recent research on the metabolism, and pharmacokinetics of phenolic compounds have revealed that they can act mainly through metabolites and catabolites formed after their intake [7]. Furthermore, various mechanisms underlying the neuroprotective effects of tannins are proposed. Since the goal of the neuroprotection approach is to limit pathological mechanisms leading to neuronal dysfunction at the molecular level such as: oxidative stress, neuroinflamation, protein aggregation, mitochondrial dysfunction and aberrant cellular signalling [8], herein, this mini-review outlines current knowledge about potential diverse neuroprotective abilities of gallotannins present in different plant preparations (mostly extracts) that are relevant to the mechanisms.

\section{Evidences for neuroprotective proper- ties of gallotannin and its derivatives}

Since oxidative damage to neuronal micro-organelles or cell bodies, accumulation of iron ion species and a decrease in the cellular antioxidant pool in the brain play a pivotal role in pathophysiology of neurodegeneration, antioxidant properties of tannins appears to be beneficial for neuroprotection [6; 9]. However, it is suggested that due to low, physiological concentrations polyphenols in brain, including tannins, act rather as "indirect" antioxidants by modulating the activity of antioxidant enzymes [6; 10]. Results of several studies gave directions to the coming to that thesis. Polyphenols have been shown to interact with critical neuronal/ glial intracellular signalling pathways involved in memory, neuronal differentiation and neuronal resistance to neurotoxins, including oxidants and inflammatory mediators [10]. Therefore, several experiments have been conducted aimed at demonstrating these neuroprotective properties for tannins $[6,10,11]$. A protective effect of water extract of Uncaria sinensis (OLIV.) HAVIL., a main medicinal plant composing Choto-san - a Kampo (traditional medicine of Japan) formula (Diao-Teng-San in Chinese) (consisting of: Atractylodes lancea rhizome, Poria sclerotium, Cnidium rhizome, Uncaria Hook, Japanese Angelica root, Bupleurum root, and Glycyrrhiza) [12-15], on glutamate-induced neuronal death in cultured cerebellar granule cells through the inhibition of $\mathrm{Ca} 2+$ influx was presented by Itoh et al [16]. The Uncaria sinensis (US) extract in a dose-dependent manner (at concentrations of 10(-5) to $10(-4) \mathrm{g} / \mathrm{ml}$ ) caused a significant protective effect against glutamate-induced cell death of cultured cerebellar granule cells compared to exposure to glutamate only. In a dose-dependent manner it blocked of the $\mathrm{Ca} 2+$ influx into cells by glutamate [16]. These results prompted researchers to investigate whether identified in $U$. sinensis extract tannin compound possess neuroprotective activities. Furthermore, an evidence of neuroprotective property of epicatechin, catechin, procyanidin B-1, procyanidin B-2, hyperin and caffeic acid isolated from the hooks and stems of Uncaria sinensis (HSUS) via protection against glutamate-induced neuronal death in cultured cerebellar granule cells by inhibition of $\mathrm{Ca} 2+$ influx was provided by Shimada et al. It was shown that the treatment with epicatechin (100$300 \mathrm{uM})$, catechin (300 uM), procyanidin B-1 (30-300 $\mathrm{uM}$ ) and procyanidin B-2 (100-300 uM) caused a significant increase of cells viability and inhibition of $\mathrm{Ca} 2+$ influx into cells induced by glutamate [17]

Early in vitro cell-free studies and cellular assays have revealed that gallotannin, a complex mixture of tannins purified from oak gall, has been shown to inhibit PARG (PARP (poly(ADP-ribose) glycohydrolase - a key enzyme degrading ADP-ribose polymers) activity [18;19]. It was also shown that it significantly reduced oxidative ( $\mathrm{H} 2 \mathrm{O} 2)$-induced cell death (after 24 and $72 \mathrm{~h}$ of $\mathrm{H} 2 \mathrm{O} 2$ exposure; $100 \mathrm{nM}$ of gallotannin) in murine astrocytes cell culture with 10 -fold more potent activity than the PARG inhibitor benzamide in preventing such process [20]. Another study by Ying et al [21] revealed that gallotannin and nobotanin $B$ (another gallotannin) equally decreased PARG (PARP1) proteins in mouse and astrocytes cell cultures exposed to hydrogen peroxide or $\mathrm{N}$-methyl-d-aspartate (NMDA), the DNA alkylating agent, and N-methyl-N'-nitro-N-nitrosoguanidine (MNNG) compared to reference benzamide causing their marked reduction. Gallotannin and benzamide both prevented the $\mathrm{NAD}(+)$ depletion resulting from PARP1 activation by MNNG or $\mathrm{H}(2) \mathrm{O}(2)$, with opposite effects on protein poly(ADP-ribosyl)ation. In this case benzamide decreased, while the gallotannin showed tendency to increase of poly(ADP-ribose) proteins accumulation during MNNG exposure in neuron cultures. Thus, results obtained by Ying et al. suggest that PARG inhibitors do not inhibit PARP1 directly, but instead prevent PARP1-mediated cell death by slowing the turnover of poly(ADP-ribose) and thus slowing $\mathrm{NAD}(+)$ consumption. One possible explanation for why gallotannin treatment leads to an apparent accumulation of PARG proteins may be it unspecific binding to biomolecules and protein staining [21].

Another, a complex experiment giving the evidence that gallotannin is an inhibitor of PARG was made by Falsig et al., however the result showed that such activ- 
ity is not specific, and that it rather does not work in cells [22]. For this purpose a comparison of the PARG inhibitory activities between tannic acid, gallotannin and compound N-bis-(3-phenyl-propyl)-9-oxo-fluorene-2,7-diamide (GPI 16552 - a potentially specific PARG inhibitor) was made in an in vitro cell-free experiment and in three PARP-1-dependent cell death (murine fetal astrocytes) models plus one inflammation model in primary astrocytes [22]. Moreover, an ability of gallotannin to inhibit recombinant human PARG was also examined. It was found that it was indeed an inhibitor of PARG as previously shown. However, results from these experiments showed that gallotannin (neither the reference inhibitor - GPI 16552) in conducted experimental cellular death models not fully effectively inhibited PARG activity. The IC50 of gallotannin in a PARG assay based on a cellular lysate was increased to about $150 \mathrm{uM}$. In primary astrocytes studied gallotannin dose dependently (10 - 50uM; 30 min exposition) blocked almost completely all cell death in the $\mathrm{H} 2 \mathrm{O} 2$ and SIN-1 models, but in the MNNG model did not rescue cells it enhanced cell death in a concentration-dependent fashion. Its action in this case was different from that observed in the case of GPI 16552 which did not gave any effect in MNNG model (in the concentration range $20,40,60,80,100,120,140 \mathrm{uM}$ ), even at highest concentrations (in SIN-1-induced cell death a small effect was seen at high GPI concentrations). This effect could be due to strong unspecific protein binding. Unspecific protein binding by gallotannin possibly also inhibited NOs as described in cell-free systems [23-25]. Authors indicated that this molecule might apparently "inhibit PARG", because it causes DNA strand breaks that activate PARP [26]. Such quite strong antioxidant effects of gallotannin against $\mathrm{H} 2 \mathrm{O} 2$ could also be due to its strong iron chelating effects [27]. Further analysis revealed that two other gallotannin-similar, polyphenolic compounds - quercetin (five phenol groups and one resonating oxo group) and catechin (five phenol groups) blocked cell death in the hydrogen peroxide model at concentrations similar to gallotannin, suggesting that PARG inhibition is not necessary for cytoprotection by this tannin only. Authors statement that its ability to effectively penetrate the cell membrane may be questionable should be here highlighted [22].

In H2O2-activated cells a decrease of PAR staining was observed and was in opposite of what was previously reported and inconsistent with PARG inhibition [28]. If gallotannin acted as a PARG inhibitor, a delay in the decay of PAR, and thus a prolonged PAR accumulation would be expected, which was not observed.
Studied gallotannin (similarly to GPI-16552) after exposure of astrocytes with $1 \mathrm{mM}$ of $\mathrm{H} 2 \mathrm{O} 2$ did not enhanced the staining for PAR at any time point of experiment duration (0 - $30 \mathrm{~min}$ ) which may suggest its unselective PARG inhibition due to the fact that, according to authors, a selective PARG inhibitor is expected to enhance PAR accumulation and/or delayed the time-dependent loss PAR presence. A possible inhibition of PARP was also visible in HeLa cells at concentration of gallotannin above $100 \mathrm{uM}$ which caused a significant increase of PAR accumulation [28]. And this effect from experiment in HeLA cells was opposite to results from Ying et al [21]. According to the authors, it is not excluded that the activity of other cellular PAR-hydrolysing enzymes could be responsible for the lack of a PAR accumulation in cells treated with specific PARG inhibitors $[22,28]$. In sum, observed protective effects from $\mathrm{H} 2 \mathrm{O} 2$ at 5-10 uM, i.e., 10- to 15-fold below the IC50 values of gallotannin in cell extracts again suggested that gallotannin does not protect due to PARG inhibition [28].

A significant anti-inflammatory potential of gallotannin correlated with its antioxidant properties was also emphasized in cell-free conditions (concentrations: $0 ; 1 ; 5 ; 10 ; 20 \mathrm{uM}$ ) and in a validated model of primary astrocyte inflammation (stimulated with a complex mixture of proinflammatory cytokines (CCM) containing TNF-a, interleukin-1h, and IFN-g) involving the production of NO [22]. In this experiment gallotannin blocked the accumulation of nitrite (concentrations: $0 ; 2.5 ; 5 ; 7.5 ; 10 \mathrm{uM}$ ) to a similar extent and with a concentration-dependent manner, thus it acted as a scavenger of NO without ever interfering with cellular processes. Interestingly, this inhibition was not due to transcriptional or translational inhibition, because gallotannin rather increased the levels of iNOS protein [22]. Further in vitro (non-cellular) experiments also indicated the ability of studied gallotannin to inhibition of PARG (PAR degradation) (almost completely at 5-10 UM concentration) which suggest that this compound was indeed a potent in vitro inhibitor of PARG. This effect was even stronger than observed in the case of reference GPI-16552 [19]. Based on above studies it was concluded that gallotannin is a PARG inhibitor in cell-free assays and acts as a strong antioxidant that can protect cells from oxidative stress. Moreover, high concentrations of gallotannin may be required to inhibit PARG in complex biological system. However, the concentration used in the cell lysates-based PAR accumulation assays would cause massive cell death in other cellular system. Authors hypothesized also 
that due to the fact that no effect in intact cells and no penetration in studied monolayer was detected it could be suggested that gallotannin is cell impermeable and mediates its effect extracellularly by lowering the concentrations of reactive oxygen species, and therefore its activity can be linked to the extracellular oxidative system. However, so far, no evidence suggesting its penetration through the cellular monolayer were found [22]. Hence, the biological activity of gallotannin in cells is still the subject of scientific inquiry.

It is also suggested that biological activity of tannins is strongly dependent on number of gallated rings. Choi et al [29] revealed that the 1,2,3,4,6-Penta-0-galloyl-beta-D-glucose (PGG) (10-50 uM), a major component of the crude Paeonia suffruticosa ANDREWS root (Ranunculaceae), was able to protect neuronal Neuro 2A cells from oxidative stress via the significant, concentration- and time-dependent induction of $\mathrm{HO}-1$ (heme oxygenase 1; an inducible stress protein that degrades heme to the neuroactive molecule, carbon monoxide and the anti-oxidant, biliverdin) gene expression and its activity. Pretreatment of these cells with PGG resulted in enhanced cellular resistance to hydrogen peroxide [29]. Tan et al [30] demonstrated that several gallotannins (1,2,3-tri-0-galloyl- $\beta$-D-glucose; 1,4,6tri-0-galloyl- $\beta$-D-glucose; $3,4,6$-tri-0-galloyl-D-glucose; 1,2,3,6-tetra-0-galloyl- $\beta$-D-glucose; 1,2,3,4,6penta-0-galloyl- $\beta$-D-glucose (PGG); 3,6-di-0-galloyl-D-glucose), among others bio-active compounds like galloylated cyanogenic glycosides, isolated from the leaves (ethyl acetate extract or aqueous extracts, respectively) of Phyllagathis rotundifolia (Melastomataceae) exhibited remarkable neuroprotective activities against oxidative damage in vitro in neuroblastoma-glioma hybrid NG108-15 cells as compared to galloylated cyanogenic glucosides and ellagic acid derivatives in a dose-dependent manner. Analyzed gallotannins also increased the neuroblastoma-glioma hybrid cell viability in a dose dependent manner. The compound PGG and 1,2,3,6- tetra-0-galloyl- $\beta$-D-glucose significantly inhibited $\mathrm{H} 2 \mathrm{O} 2$-induced neuron cells damage in a dose-dependent manner at concentrations of $6.25-100 \mu \mathrm{M}$. The inhibitory activity of 1,2,3,6tetra-0-galloyl- $\beta$-D-glucose was comparable to that of catechin. However, the neuroprotective activity of PGG was more potent than that of catechin [30]. This compound has also been reported to not only increase the cellular resistance to $\mathrm{H} 2 \mathrm{O} 2$ but also highly protected neuronal cells from $\mathrm{H} 2 \mathrm{O} 2$-induction damage via induction of $\mathrm{HO}-1$ gene expression [29,30]. According to Warden et al., the level of galloylated catechins in human urine after black tea consumption was 10-fold lower than that of non-galloylated catechins, which strongly indicates that galloylation increases resorption of catechins [31].

Among many different polyphenol subgroups, gallotannins have been relatively poorly examined in the context of the anti-amyloidogenic activities. Only few publications have appeared in recent years documenting their strong neuroprotective abilities. It has been shown, for example, that PGG exhibited a strong anti-aggregation effect on $\beta$-amyloid in Alzheimer disease [32]. An in vitro SK-N-SH cell line experiment demonstrated that PGG isolated from Paeonia suffruticosa at the $3 \mu \mathrm{M}$ concentration inhibits $A \beta 1-42$ fibrils formation of over $50 \%$, while the $100 \mu \mathrm{M}$ concentration completely inhibits formation of $A \beta 1-42$ fibrils. Fujiwara and co-workers have also demonstrated that PGG oral administration to mice Tg2576 APPswe race 8 $\mathrm{mg} / \mathrm{kg} /$ day strongly decreased level of $A \beta 1-40$ aggregates (from $4000 \mathrm{pmol} / \mathrm{g}$ brain to about $2500 \mathrm{pmol} / \mathrm{g}$ brain). In the case of aggregates of $A \beta 1-42$ the dose was approximately $100 \mathrm{pmol} / \mathrm{g}$ brain in the control and about $50 \mathrm{pmol} / \mathrm{g}$ brain in animals used in studies [32]. Another compound - tannic acid - turned out to be a natural $\beta$-secretase inhibitor that prevented cognitive impairment and mitigates AD pathology in PS/APP transgenic mice. In addition, it reduced the effects of Alzheimer's like neuropathology in mice overproducing $A \beta 1-40$ and $A \beta$ 1-42 [33]. Another in vivo studies by Hartman et al. showed that in other transgenic mice (strain $\mathrm{APP}_{\mathrm{Sw}} / \mathrm{Tg} 2576$ ) who drank the pomegranate juice (containing 115 ppm of ellagic acid, 5 ppm of gallic acid, 1880 ppm of hydrolysable tannins; among them: gallotannins, ellagitannins, punicalagin and $369 \mathrm{ppm}$ of anthocyanins and their glucosides) the level of $A \beta$ plaques and fibrils in both the hippocampus and cortex dorsal has been decreased [34]. Determining which of these compounds showed these properties and with which intensity requires further complex research.

\section{Conclusions}

Summarizing, data presented in this manuscript provide some evidences about the neuroprotective potential of gallotannins. However, their mechanism of actions remains still not fully understood. Therefore, it is highly justified to further explore the mechanism of this class of natural-origin protective agents against neurodegeneration. The complex knowledge about their polypharmacological activities may be of significance for neuroprotection. 
Acknowledgements

\section{Conflict of interest statement}

The authors declare no conflict of interest.

\section{Funding sources}

There are no sources of funding to declare.

\section{References}

1. Rodriguez-Mateos A, Vauzour D, Krueger CG, Shanmuganayagam D, Reed J, Calani L, Mena P, Del Rio D, Crozier A. Bioavailability, bioactivity and impact on health of dietary flavonoids and related compounds: an update. Arch Toxicol. 2014;88(10):1803-53.

2. Del Rio D, Rodriguez-Mateos A, Spencer JP, Tognolini M, Borges G, Crozier A. Dietary (poly)phenolics in human health: structures, bioavailability, and evidence of protective effects against chronic diseases. Antioxid Redox Signal. 2013;18(14):1818-92.

3. Tejada S, Setzer W, Daglia M et al. Neuroprotective effects of Ellagitannins: A brief review. Curr Drug Targets. 2016 [Epub ahead of print].

4. Gong YS, Guo J, Hu K, Gao YQ, Xie BJ, Sun ZD, Yang EN, Hou FL. Ameliorative effect of lotus seedpod proanthocyanidins on cognitive impairment and brain aging induced by D-galactose. Exp Gerontol. 2016;74:21-8.

5. Smeriglio A, Barreca D, Bellocco E, Trombetta D. Proanthocyanidins and hydrolysable tannins: occurrence, dietary intake and pharmacological effects. $\mathrm{Br} \mathrm{J}$ Pharmacol. 2016 Sep 20. doi: 10.1111/bph.13630. [Epub ahead of print].

6. Ebrahimi A, Schluesener $H$. Natural polyphenols against neurodegenerative disorders: potentials and pitfalls. Ageing Res Rev. 2012;11:329-345.

7. Tomás-Barberán FA, González-Sarrías A, García-Villalba $\mathrm{R}$ et al. Urolithins, the rescue of 'old' metabolites to understand a 'new' concept: metabotypes as a nexus between phenolic metabolism, microbiota dysbiosis and host health status. Mol Nutr Food Res. 2016. doi: 10.1002/mnfr.201500901. [Epub ahead of print].

8. Caruana M, Cauchi R, Vassallo N. Putative Role of Red Wine Polyphenols against Brain Pathology in Alzheimer's and Parkinson's Disease. Front Nutr. 2016;12:3-31.

9. Spencer JP, Vafeiadou K, Williams RJ, et. al. Neuroinflammation: modulation by flavonoids and mechanisms of action. Mol Aspects Med. 2012;33:83-97.

10. Williams RJ, Spencer JP. Flavonoids, cognition, and dementia: actions, mechanisms, and potential therapeutic utility for Alzheimer disease. Free Radic Biol Med. 2012;52:35-45.

11. Mandel SA, Amit T, Kalfon L, Reznichenko L, Weinreb O, Youdim MB. Cell signaling pathways and iron chelation in the neurorestorative activity of green tea polyphenols: special reference to epigallocatechin gallate (EGCG). J Alzheimers Dis. 2008;15(2):211-22.

12. Terasawa K, Shimada Y, Kita T, Yamamoto T, Tosa H, Tanaka N, Saito Y, Kanaki E, Goto S, Mizushima N, Fujioka M, Takase S, Seki H, Kimura I, Ogawa T,Nakamura S, Araki G, Maruyama I, Maruyama Y, Takaori S. Choto-san in the treatment of vascular dementia: a double-blind, placebo-controlled study. Phytomedicine. 1997;4(1):15-22.
13. Murakami Y, Zhao Q, Harada K, Tohda M, Watanabe H, Matsumoto K. Choto-san, a Kampo formula, improves chronic cerebral hypoperfusion-induced spatial learning deficit via stimulation of muscarinic M1 receptor. Pharmacol Biochem Behav. 2005;81(3):616-25.

14. Kanno H, Kawakami Z, Tabuchi M, Mizoguchi K, Ikarashi $Y$, Kase Y. Protective effects of glycycoumarin and procyanidin B1, active components of traditional Japanese medicine yokukansan, on amyloid $\beta$ oligomer-induced neuronal death. J Ethnopharmacol. 2015;159:122-8.

15. Ikarashi Y, Mizoguchi K. Neuropharmacological efficacy of the traditional Japanese Kampo medicine yokukansan and its active ingredients. Pharmacol Ther. 2016;166:84-95.

16. Itoh T, Shimada Y, Terasawa K. Efficacy of Choto-san on vascular dementia and the protective effect of the hooks and stems of Uncaria sinensis on glutamate-induced neuronal death. Mech Ageing Dev. 1999;111(2-3):15573.

17. Shimada Y, Goto H, Kogure T, Shibahara N, Sakakibara I, Sasaki H, Terasawa K. Protective effect of phenolic compounds isolated from the hooks and stems of Uncaria sinensis on glutamate-induced neuronal death. Am J Chin Med. 2001;29(1):173-80.

18. Tsai $Y J$, Aoki $T$, Maruta $H$, Abe $H$, Sakagami $H$, Hatano T, Okuda T, Tanuma S. Mouse mammary tumor virus gene expression is suppressed by oligomeric ellagitannins, novel inhibitors of poly(ADPribose) glycohydrolase. J Biol Chem. 1992;267:14436-14442.

19. Aoki $K$, Nishimura $K$, Abe $H$, Maruta $H$, Sakagami $H$, Hatano T, Okuda T, Yoshida T, Tsai YJ, Uchiumi F. Novel inhibitors of poly(ADP-ribose) glycohydrolase. Biochim Biophys Acta. 1993;1158:251-256.

20. Ying W, Swanson RA. The poly(ADP-ribose) glycohydrolase inhibitor gallotannin blocks oxidative astrocyte death. Neuroreport. 2000;11(7):1385-8.

21. Ying W, Sevigny MB, Chen Y, Swanson RA. Poly(ADP-ribose) glycohydrolase mediates oxidative and excitotoxic neuronal death. Proc Natl Acad Sci USA. 2001;98(21):12227-32.

22. Falsig J, Christiansen $S H$, Feuerhahn $S$, Bürkle A, Oei $\mathrm{SL}$, Keil C, Leist M. Poly(ADP-ribose) glycohydrolase as a target for neuroprotective intervention: assessment of currently available pharmacological tools. Eur J Pharmacol. 2004;497(1):7-16.

23. Chiesi M, Schwaller, R. Inhibition of constitutive endothelial NO synthase activity by tannin and quercetin. Biochem. Pharmacol. 1995;49:495-501.

24. Kaneko $M$, Saito $Y$, Saito $H$, Matsumoto $T$, Matsuda Y, Vaught JL, Dionne CA, Angeles TS, Glicksman MA, Neff NT, Rotella DP, Kauer JC, Mallamo JP, Hudkins RL, Murakata C. Neurotrophic 3,9-bis[(alkylthio)methyl]and-bis(alkoxymethyl)-K-252a derivatives. J Med Chem. 1997; 40:1863-1869.

25. Ha, HC, Hester, LD, Snyder, SH. Poly(ADP-ribose) polymerase-1 dependence of stress-induced transcription factors and associated gene expression in glia. Proc Natl Acad Sci USA. 2002;99:3270-3275.

26. Labieniec M, Gabryelak T, Falcioni G. Antioxidant and prooxidant effects of tannins in digestive cells of the freshwater mussel Unio tumidus. Mutat Res. 2003;539:19-28. 
27. Lopes GK, Schulman HM, Hermes-Lima M. Polyphenol tannic acid inhibits hydroxyl radical formation from Fenton reaction by complexing ferrous ions. Biochim Biophys Acta. 1999;1472:142-152.

28. Keil C, Petermann E, Oei, SL. Tannins elevate the level of poly(ADP-ribose) in HeLa cell extracts. Arch Biochem Biophys. 2004;425:115-121.

29. Choi BM, Kim HJ, Oh GS, Pae HO, Oh H, Jeong $S$, Kwon TO, Kim YM, Chung HT. 1,2,3,4,6-Penta-O-galloyl-beta-D-glucose protects rat neuronal cells (Neuro $2 \mathrm{~A}$ ) from hydrogen peroxide-mediated cell death via the induction of heme oxygenase-1. Neurosci Lett. 2002;328(2):185-9.

30. Tan HP, Wong DZ, Ling SK, Chuah CH, Kadir HA. Neuroprotective activity of galloylated cyanogenic glucosides and hydrolysable tannins isolated from leaves of Phyllagathis rotundifolia. Fitoterapia. 2012;83(1):223-9.

31. Warden BA, Smith LS, Beecher GR, Balentine DA, Clevidence BA. Catechins are bioavailable in men and women drinking black tea throughout the day. J Nutr. 2001;131:1731-1737.

32. Fujiwara H, Tabuchi M, Yamaguchi T, Iwasaki K, Furukawa K, Sekiguchi K, Ikarashi Y, Kudo Y, Higuchi M, Saido TC, Maeda S, Takashima A, Hara M, Yaegashi N, Kase $\mathrm{Y}$, Arai $\mathrm{H}$. A traditional medicinal herb Paeonia suffruticosa and its active constituent 1,2,3,4,6-penta-0-galloyl-beta-D-glucopyranose have potent anti-aggrega- tion effects on Alzheimer's amyloid beta proteins in vitro and in vivo. J Neurochem. 2009;109(6):1648-57.

33. Mori T, Rezai-Zadeh K, Koyama N, Arendash GW, Yamaguchi H, Kakuda N, Horikoshi-Sakuraba Y, Tan J, Town T. Tannic acid is a natural $\beta$-secretase inhibitor that prevents cognitive impairment and mitigates Alzheimer-like pathology in transgenic mice. J Biol Chem. 2012;287(9):6912-27.

34. Hartman RE, Shah A, Fagan AM, Schwetye KE, Parsadanian M, Schulman RN, Finn MB, Holtzman DM. Pomegranate juice decreases amyloid load and improves behavior in a mouse model of Alzheimer'sdisease. Neurobiol Dis. 2006;24(3):506-15.

Acceptance for editing: 2016-12-10 Acceptance for publication: 2016-12-22

\section{Correspondence address:}

Radosław Kujawski

Department of Pharmacology

Poznan University of Medical Sciences, Poland email: radkuj@ump.edu.pl 